\title{
Development of Label-Free Impedimetric Hcg-Immunosensor Using Screen-Printed Electrode
}

\author{
Truong TN Lien ${ }^{1,2 *}$, Nguyen Xuan Viet', Miyuki Chikae', Yoshiaki Ukita' and Yuzuru Takamura' \\ ${ }^{1}$ School of Materials Science, Japan Advanced Institute of Science and Technology (JAIST), 1-1 Asahidai, Nomi, Ishikawa, 923-1292, Japan \\ ${ }^{2}$ Department of Electronic Materials, Hanoi University of Science and Technology (HUST), No. 1 Dai Co Viet, Hai Ba Trung, Hanoi, Vietnam
}

\begin{abstract}
Screen-printing (thick-film) technology is well identified as a reliable technique for fabrication of electrodes which can be used as transducer in biosensor, with several advantages including low cost, design flexibility, process automation, good reproducibility and a wide choice of materials. However, the immobilization of antibody molecules is a decisive factor for successful fabrication of immunosensors. Besides, the ability to measure human Chorionic Gonadotropin (hCG) is important in establishing the diagnosis of gestational trophoblastic disease and germ cell tumors. Moreover, Electrochemical Impedance Spectroscopy (EIS) recently has been being chosen as a main detection method because it is label-free, less destructive to the activities of biomolecule and very sensitive with comparable detection limits as optical-based sensor. In this work, a sensitive label-free impedimetric hCG-immunosensor was constructed by using a commercial screen-printing carbon ink electrode (namely Disposable Electrochemical Printed chip) as a basis. The hCG antibody was immobilized via the entrapment technique on the carbon ink electrode of DEP chip using functional molecule, 1-pyrenebutanoic acid, succinimidyl ester. The experimental results exposed that the designed immunosensor is more sensitive than other previously reported immunosensors, in the case of detection limit and linear range for antigen detection. With optimal fabrication parameters, the detection limit for a-hCG was $33 \mathrm{pg} / \mathrm{mL}$ in $10 \mathrm{mM}$ phosphate buffer saline (PBS) solution containing $1 \%$ bovine serum albumine (BSA). Furthermore, the use of inexpensive DEP chip as a basis for these immunosensors will allow simple instrumentation, disposable and portable at low cost. This work also demonstrates a new approach to develop a sensitive and labelfree impedimetric immunosensor based on screen-printed electrode for applications in clinical diagnosis.
\end{abstract}

Keywords: Human Chorionic Gonadotropin (hCG); Label-free impedimetric immunosensor; Electrochemical impedance spectroscopy (EIS); Screen-Printed Electrode; DEP chip

\section{Introduction}

Screen-printing (thick-film) technology is well identified as a reliable technique for fabrication of electrodes which can be used as transducer in biosensor with several advantages, including low cost, design flexibility, process automation, good reproducibility and a wide choice of materials [18]. Thus, it has been pursued as an alternative method for production of modern biosensors which can be incorporated in portable systems. Up to now, the biosensors based on screen-printed electrodes have increased in the many areas of bioanalytical chemistry, analysis mutant genes, and clinical diagnostic for health care and environment $[3,8,22]$. Additional, a wide range of biomolecular recognition elements such as enzymes, antibodies, or micro-organisms has been used to develop these screen-printed biosensors [15]. Antibody-based biosensors (immunosensors) are more sensitive and selective than enzyme-based biosensors because the antibodies can be bound specifically to an analyte via affinity coupling. Unlike enzyme-based biosensors where either the co-substrate or the product of an enzyme reaction is monitored, antibody-based biosensors detect antigen or antibody concentration either by direct changes in the transducer output resulting from the binding event, or by means of indirect competitive and displacement reactions using optical, piezoelectric, or electrochemical techniques. In many cases, this results in very low detection limits for immunosensor assays [16]. However, the immobilization of antibody molecules is a decisive factor for successful fabrication of immunosensors. The immobilization method must maintain the activity and enhanced stability of biomolecules and it is controllable over the distribution and orientation of the immobilized species. The methods that are typically used including the physical absorption, chemical cross-linking and entrapment. Among them, biomolecule immobilization method by entrapment within a suitable matrix which then deposited on the screen-printed support can improve the stability of the biorecognition component [18]. The immobilization matrices used include gels, polymers, pastes, or ink. Normally, the biological material is mixed and well homogenized with the supporting material followed by being applied over the electrode as an additional membrane and then dried or polymerized. Human Chorionic Gonadotropin (hCG) is glycoprotein composed of 244 amino acids with a molecular mass of $36.7 \mathrm{kDa}$. Its most important uses as a tumor marker are in gestational trophoblastic disease and germ cell tumors. Measurement of hCG is important in establishing the diagnosis of the disease. 3 Besides, electrochemical impedance spectroscopy (EIS) recently has been being chosen as a main detection method because it has some important advantages over number of electrochemical methods such as amperometry and potentiometry. This sensor is label-free with a direct detection of specific binding event, less destructive to the activities of biomolecule due to the small voltage excitation during detection, simple operation and very sensitive with comparable detection limits as optical-based sensor [5]. Moreover, the use of EIS as a detection method to quantitate antigen has been recently reported with detection limit in the ng.mL-1 to pg.mL-1 range

*Corresponding author: Truong TN Lien, Department of Electronic Materials Hanoi University of Science and Technology, Vietnam, E-mail: truongtnlien@gmail. com

Received July 07, 2011; Accepted August 28, 2011; Published September 03 2011

Citation: Lien TTN, Viet NX, Chikae M, Ukita Y, Takamura Y (2011) Development of Label-Free Impedimetric Hcg-Immunosensor Using Screen-Printed Electrode. J Biosens Bioelectron 2:107. doi:10.4172/2155-6210.1000107

Copyright: @ 2011 Lien TTN, et al. This is an open-access article distributed under the terms of the Creative Commons Attribution License, which permits unrestricted use, distribution, and reproduction in any medium, provided the original author and source are credited. 
Citation: Lien TTN, Viet NX, Chikae M, Ukita Y, Takamura Y (2011) Development of Label-Free Impedimetric Hcg-Immunosensor Using ScreenPrinted Electrode. J Biosens Bioelectron 2:107. doi:10.4172/2155-6210.1000107

Page 2 of 6

$[1,2,7,13,20]$. In this work, we developed a sensitive faradaic impedimetric immunosensor utilizing commercial screen-printing carbon ink electrode (namely Disposable Electrochemical Printed chip) as the basis for $a$-hCG detection. In addition, a simple and general approach to noncovalent functionalization of carbon electrode surface, which will be used to immobilize antibody with high degree of control and specific, is also presented. The noncovalent functionalization involves a functional molecule, 1-pyrenebutanoic acid succinimidyl ester. Following this, the EIS technique was applied to monitor the formation of the recognition of hCG antibody onto carbon ink electrode as well as the hCG antibody- antigen interaction.

\section{Experimental}

\section{Reagents and apparatus}

1-pyrenebutanoic acid, succinimidyl ester (1) was supplied from Eugene, Oregon (USA). Ethanolamine, Bovine Serum Albumine (BSA) and Dimethyl Sulfoxide Dehydrated (DMSO) were purchased from Sigma Aldrich. The human Chorionic Gonadotropin (hCG) monoclonal antibody (Mab) and $a-h C G$ were supplied by Medix Biochemica (Finland). All reagents used were of the analytical grade or the highest commercially available purity and used as supplied without further purification. All solutions were prepared with deionized water of resistivity no less than $18 \mathrm{M} \Omega \mathrm{cm}$. The commercial Disposable Electrochemical Printed (DEP) chips were obtained from BioDevice Technology Ltd., Japan (http://www.biodevicetech.com). The chips were fabricated by screen-printing technology and designed as system with three electrodes containing carbon ink working, carbon ink counter and $\mathrm{Ag} /$ $\mathrm{AgCl}$ ink reference electrodes. The carbon ink contained 75\% (w/w) graphite powder and $25 \%(\mathrm{w} / \mathrm{w})$ mineral oil (Sigma). Surface area of the working electrode is $2.64 \mathrm{~mm} 2$. The structure of DEP chip used within this work is shown in Figure 1. An AutoLab PGSTAT 30 system (EcoChemie B.V., Ultrecht, The Netherlands) was used to perform EIS measurements.

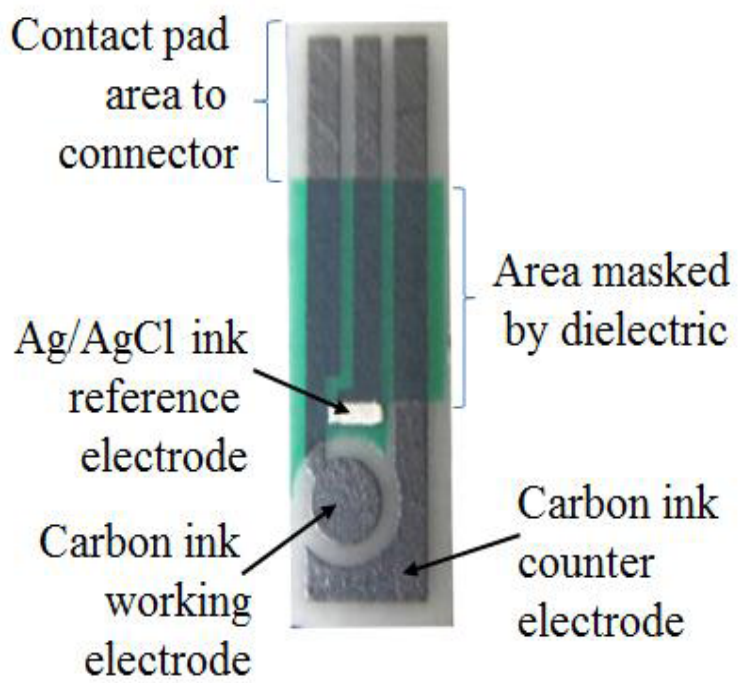

Figure 1: The structure of commercial Disposable Electrochemical printed (DEP) chip used within this work. The chip were fabricated by screen-printing technology and designed as system with three electrodes.

\section{Preparation of 1 - Mab hCG conjugated}

The Mab hCG at $200 \mu \mathrm{g} / \mathrm{mL}$ of concentration was prepared by diluting in $10 \mathrm{mM}$ carbonate buffer $(\mathrm{pH}=8.2) .1$ was dissolved in DMSO with $10 \mathrm{mg} / \mathrm{mL}$ concentration. Then, $100 \mu \mathrm{L}$ of 1 was added into $1 \mathrm{~mL}$ of diluted Mab hCG and mixed by shaker at room temperature for 3 hours. In this step, the amine groups on a protein react with the anchored succinimidyl ester to form amide bonds for protein conjugation. The prepared 1-Mab hCG complex solution was centrifuged at $15,000 \mathrm{rpm}$ for $20 \mathrm{~min}$ at $4^{\circ} \mathrm{C}$ in centrifuge using millipore with $200 \mathrm{~nm}$ in diameter. After centrifuge, this 1-Mab hCG conjugated solution was stored at $4^{\circ} \mathrm{C}$ for further experiments.

\section{Immunosensor fabrication}

The immunosensors were fabricated by using two different methods, which are named method A and method B.

\section{Method A}

There are three steps in this method (Figure 2a). Firstly, (1) was dissolved in solution containing $70 \%$ of DMSO and $30 \%$ of deionized water with $1 \mathrm{mg} / \mathrm{mL}$ of concentration. A volume of $2 \mu \mathrm{L}$ of this solution was dropped onto carbon ink working electrode of DEP chip for 1 hour followed by rinsing several times with deionized water to wash away excess reagent and then dried over a stream $\mathrm{N}_{2}$ gas. In this step, the pyrenyl groups interacted strongly with the basal plane of carbon graphite via $\pi$-stacking $[9,11]$ and provided a fixation point for $(1)$ on this surface. The anchored molecules of 1 on the carbon surface are highly stable against desorption in aqueous solution. This leads to the functionalization of carbon surface with succinimidyl ester groups that are highly reactive to nucleophilic substitution by primary and secondary amines that exist in abundance on the surface of most proteins [19]. After that, $2 \mu \mathrm{L}$ of $200 \mu \mathrm{g} / \mathrm{mL}$ Mab hCG was placed onto surface of these electrodes for 1 hour at room temperature followed by further washing with $10 \mathrm{mM}$ PBS solution containing $0.05 \%$ Tween 20 to remove the loosely bound antibodies and then drying over a gentle stream N2 gas. In this step, the hCG antibody was successfully immobilized onto carbon electrodes via the attachment of amine groups of antibody with succinimidyl ester groups on functionalized electrode by (1). Finally, the Mab hCG-modified electrodes were subjected to $2 \mu \mathrm{L}$ of BSA ( $1 \%$ in $10 \mathrm{mM} \mathrm{PBS)}$ and incubated at $4^{\circ} \mathrm{C}$ for 15 hours for blocking the nonspecific binding. Following this, the electrodes also were rinsed $10 \mathrm{mM}$ PBS solution containing $0.05 \%$ Tween 20 followed by deionized water and then dried over a gentle stream $\mathrm{N}_{2}$ gas. The immunosensors (labeled immunosensor A) are ready to use at this point.

\section{Method B}

A volume of $2 \mu \mathrm{L}$ of (1) - Mab hCG conjugated solution was dropped onto the carbon ink working electrode surface of DEP chip and incubated at $40 \mathrm{C}$ for 18 hours. Afterward, the electrodes were rinsed with $10 \mathrm{~mL}$ of $10 \mathrm{mM}$ PBS solution containing $0.05 \%$ Tween 20 followed by deionized water to remove the loosely bound antibodies and dried over a stream $\mathrm{N}_{2}$ gas. Then, the hCG-modified electrode was subjected to 2 $\mu \mathrm{L}$ of $100 \mathrm{mM}$ Ethanolamine solution for 1 hour at room temperature in order to block the remaining non-specific adsorption-reactive sites. The electrode was also rinsed with $10 \mathrm{~mL}$ of $10 \mathrm{mM}$ PBS solution containing $0.05 \%$ Tween 20 followed by deionized water, then dried over a gentle stream $\mathrm{N}_{2}$ gas. The immunosensors (labeled immunosensor $\mathrm{B}$ ) can be used immediately. The Figure $2 \mathrm{~b}$ illustrates the whole process to fabricate the immunosensor $\mathrm{B}$. 
Citation: Lien TTN, Viet NX, Chikae M, Ukita Y, Takamura Y (2011) Development of Label-Free Impedimetric Hcg-Immunosensor Using ScreenPrinted Electrode. J Biosens Bioelectron 2:107. doi:10.4172/2155-6210.1000107

Page 3 of 6

\section{a-hCG detection procedure}

The $a$-hCG was suspended in 10mM PBS solution containing $1 \%$ Bovine Serum Albumine (BSA) with required antigen concentration. In this case, the range of $\alpha-h C G$ concentration from $200 \mathrm{pg} / \mathrm{mL}$ to $70 \mathrm{ng} /$ $\mathrm{mL}$ was utilized. The immunosensors were first EIS measured without $a$-hCG addition. Following this, $2 \mu \mathrm{l}$ required $\alpha$-hCG concentration was added on each immunosensor surface for $40 \mathrm{~min}$ at room temperature to let the $\alpha$-hCG attach to the Mab hCG. Then, immunosensors were rinsed with 10mM PBS followed by deionized water and dried over a gentle stream $\mathrm{N}_{2}$ gas. Finally, all immunosensors were subjected to EIS measurement. The impedance spectra was recorded in $0.1 \mathrm{M} \mathrm{KCl}$ solution containing $5 \mathrm{mM}$ of $\mathrm{K}_{3}\left[\mathrm{Fe}(\mathrm{CN})_{6}\right] / \mathrm{K}_{4}\left[\mathrm{Fe}(\mathrm{CN})_{6}\right]$ within the frequency range from $100 \mathrm{kHz}$ to $50 \mathrm{mHz}$. An ac probe amplitude of $10 \mathrm{mV}$ was applied to the system around the Open Circuit Potential (OCP).

\section{Results and Discussion}

\section{Labelless impedimetric immunosensor}

In a electrochemical impedance sensor, the detection is based on

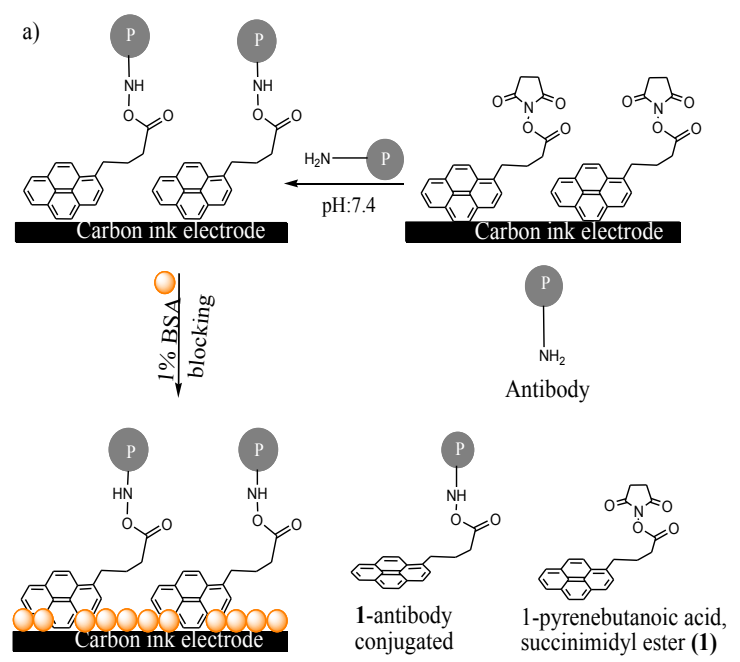

b)

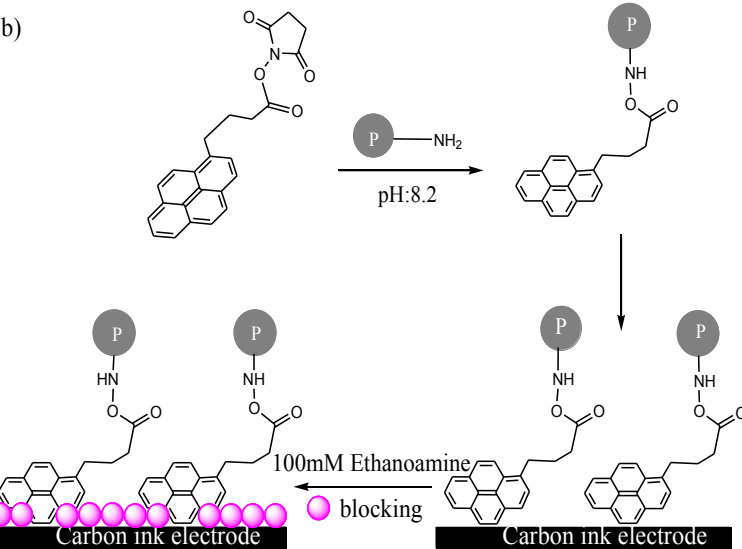

Figure 2: Schematic diagrams represent the fabrication of impedimetrichCGimmunosensors based on DEP chip following a) method $A$ and $b$ ) method $B$.In the method $A$, the carbon ink electrode of DEP chip is modified first by using a functional molecule, 1-pyrenebutanoic acid succinimidyl ester (1) and thence MabhCG immobilization via succimidyl ester groups. However, in the method $\mathrm{B}$, the MabhCG is conjugated first with 1 and then dropped directly onto carbon ink working electrode surface of DEP chip. the principle that any substance attached on its electrode will change the measured impedance. In this case, the hCG antibody receptor and the bound hCG antigen together can be considered as a coating film with expected to effect the sensor impedance signal. The impedance measurement can be performed in the absence or presence of a redox probe, which are referred to nonfaradaic and faradaic impedance measurements [4]. In the absence of a redox probe, the measured impedance signal results directly from the substances that are adherently attached to the electrode surface. In other words, the impedance is influenced by the changes in amount, growth and morphological behavior of adherent substance. In the presence of a redox probe, the sensor verifies the biological events occurring on its surface by measuring the changes in impedance spectroscopy. Therefore, this method has been considered as an efficient way to monitor the formation of antigenantibody interaction. In this work, we developed a electrochemical impedance immunosensor using a redox probe, $\left[\mathrm{Fe}(\mathrm{CN})_{6}\right]^{3-14-}$, for $a-h C G$ detection. (Figure 3) illustrates the principle of this sensor. The Mab hCG was first immobilized onto carbon electrode. Then, this modified electrode was exposed to a $\alpha-h C G$ solution. The Mab hCG receptor together with the bound $\alpha-h C G$ can be considered as a coating film with expectation to effectively block the charge (electron) transfer and thus amplify impedance signal. The behavior of the impedance sensor system can be well clarified by the Randles equivalent circuit which shown in Figure 3b. The circuit model includes the following four elements: (1) the ohmic resistance of the electrolyte Rs; (2) the Warburg impedance ZW of the electrode; (3) the double layer capacitance Cdl; and (4) the electron transfer resistance $\mathrm{R}_{\mathrm{CT}}$. Ideally, the Rs and $\mathrm{ZW}$ represent the bulk properties of the electrolyte solution and diffusion of the redox probe, whereas $\mathrm{Cdl}$ and $\mathrm{R}_{\mathrm{CT}}$ depend on the dielectric and insulting characteristics at the interface between electrode and electrolyte. They are both affected by modification occurring on the electrode surface $[4,15,17]$. Thus, $\mathrm{Cdl}$ and $\mathrm{R}_{\mathrm{CT}}$ are parameters that mainly used as signals in impedance sensor. In this case, $R_{C T}$ is chosen for sensing the interfacial properties of electrodes. The (Figure 3c) shows the Nyquist plot (Zim vs. Zre), which is best way to imagine and determine the electron transfer resistance $\mathrm{R}_{\mathrm{CT}}$. The typical Nyquist plot included a semicircle part at high frequency region corresponding to the electron transfer limited process and a linear part at lower frequencies resulting from the diffusion limiting step of the electrochemical process. Therefore, the

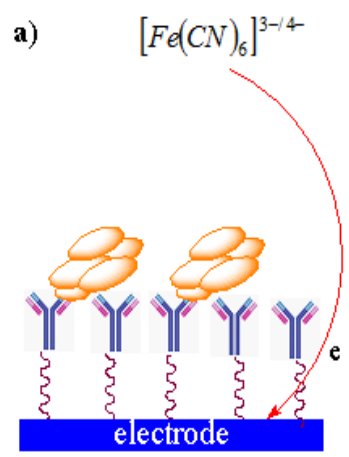

b)

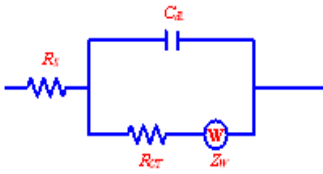

c)

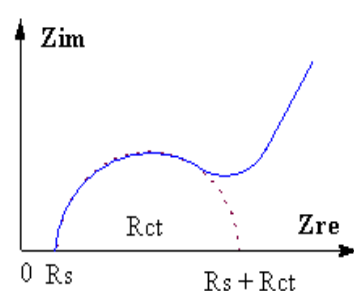

Figure 3: Schematic illustrate a) the principle of the electrochemical impedance immunosensor for antigen detection using b) the Randle's equivalent circuit to fit impedance spectroscopy by commercial software Autolab data analysis (EcoChemie) and c) typical Nyquist plot (Zim vs. Zre) of Faradaic impedance spectrum in presence of redox probe $\left[\mathrm{Fe}(\mathrm{CN})_{6}\right]^{3-14-}$. 
electron transfer kinetic parameters and diffusion characteristic can be extracted from the semicircle and linear parts of the impedance spectrum, respectively. The intercept of the semicircle with the Zre axis at high frequency is equal to $R_{s}$. The diameter of semicircle equals to electron transfer resistance $\mathrm{R}_{\mathrm{CT}}$, which denotes the blocking behavior of the electrode surface for redox probe. Therefore, the increase and decrease in this value will be exhibited exactly the assembly of electrode surface. A significant difference in the impedance spectra of Mab hCG immobilization - modified electrode and binding of a-hCG compared with bare carbon electrode were observed in the (Figure 4). In the case of immunosensor A (Figure $4 \mathrm{a}$ ), the $\mathrm{R}_{\mathrm{CT}}$ value of carbon bare electrode is $(4.50 \pm 0.14) \mathrm{k} \Omega$. However, after functionalizing carbon electrode by 1 , the diameter of semicircle in impedance spectrum drastically increases with an increase in $\mathrm{R}_{\mathrm{CT}}$ value to $(17.53 \pm 0.34) \mathrm{k} \Omega$. A noteworthy increase in $\mathrm{R}_{\mathrm{CT}}$ value to $(90.47 \pm 5.42) \mathrm{k} \Omega$ was observed in the step of Mab hCG immobilization. An increase in the charge transfer resistance value could be explained due to the generation of an insulating protein layer on electrode. This result was confirmed that the Mab hCG was successfully immobilized onto carbon electrode surface. Following this, the Mab hCG-modified electrode was exposed to $a-h C G$ with concentration of $1 \mathrm{ng} / \mathrm{mL}$ and an increase in the value of $\mathrm{R}_{\mathrm{CT}}$ to $(98.24 \pm 5.97)$ $\mathrm{k} \Omega$ was observed. This result was further confirmed the success of Mab hCG immobilization onto the electrode. Likewise, we extracted the $\mathrm{R}_{\mathrm{CT}}$ value during stepwise modification of the electrode of immunosensor $B$ by fitting impedance spectra, which shown in (Figure $4 \mathrm{~b}$ ), to Randles equivalent circuit. We observed that after the 1-Mab hCG conjugated layer is immobilized onto carbon electrode the $\mathrm{R}_{\mathrm{CT}}$ increases significantly to $(3.27 \pm 0.18) \mathrm{k} \Omega$, whereas $\mathrm{R}_{\mathrm{CT}}$ value of carbon bare electrode is $(0.72 \pm 0.02) \mathrm{k} \Omega$. A noteworthy increase in $\mathrm{R}_{\mathrm{CT}}$ value to $(6.26 \pm 0.17)$
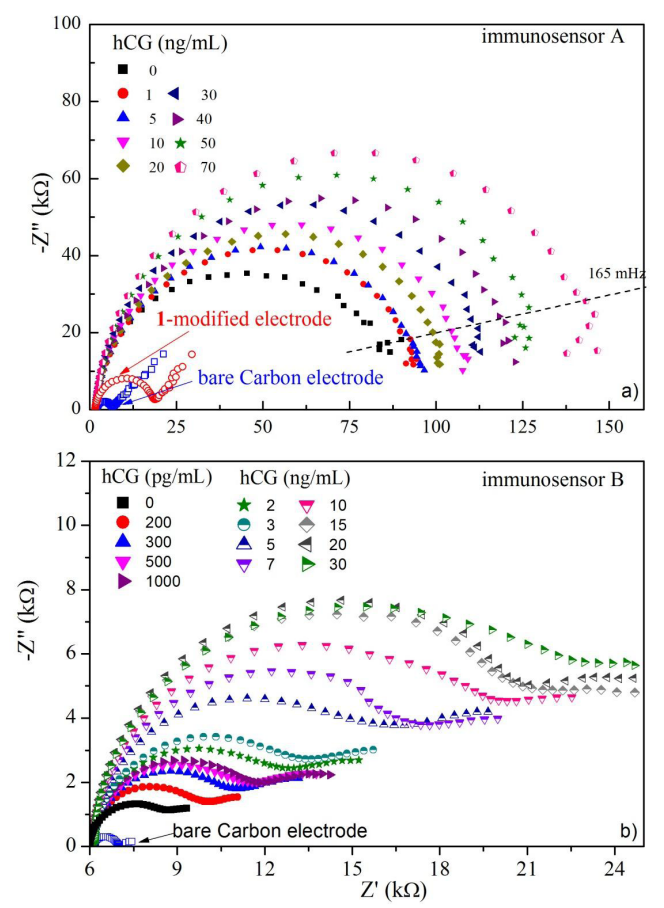

Figure 4: Impedance spectra of the electrodes of immunosensors $A$ and $B$ exposed to difference concentrations of $\alpha-h C G$. The impedance results were obtained in the solution containing $0.1 \mathrm{M} \mathrm{KCl}$ and $5 \mathrm{mMK}_{3}\left[\mathrm{Fe}(\mathrm{CN})_{6}\right] / \mathrm{K}_{4}\left[\mathrm{Fe}(\mathrm{CN})_{6}\right.$ at OCP and frequency range is from $100 \mathrm{kHz}$ to $50 \mathrm{mHz}$ with an Ac probe amplitude of $10 \mathrm{mV}$. $\mathrm{k} \Omega$ was also observed in the step of $\alpha$-hCG binding, which the 1-Mab hCG conjugated immobilization-modified electrode was exposed to $a$-hCG with concentration of $1 \mathrm{ng} / \mathrm{mL}$. This result was also confirmed the success of 1-Mab hCG conjugated immobilization onto electrode of immunosensor B.

$$
\frac{R_{C T}(M a b h C G+\alpha h C G)-R_{C T}(M a b h C G)}{R_{C T}(M a b h C G+\alpha h C G)}
$$

Besides, the percentage change of the $\mathrm{R}_{\mathrm{CT}}$ value, which is obtained up to antibody immobilization level and after $\alpha$-hCG addition is determined as:

When the immonosensor $\mathrm{A}$ and $\mathrm{B}$ are exposed to $\mathrm{a}-\mathrm{hCG}$ with concentration of $1 \mathrm{ng} / \mathrm{mL}$, the normalized percentage changes of the $\mathrm{R}_{\mathrm{CT}}$ values were $8 \%$ and $48 \%$, respectively. This suggests that the Mab hCG immobilization efficiency of the immunosensor B might higher than immunosensor A's. In other words, the sensitivity of immunosensor B could better than that of immunosensor A. The reason is that DMSO is a polar aprotic solvent with weakly acidic property. A large amount of the solvent (in method A) influences the carbon ink electrode surface due to the ability to dissolve adhesives in carbon ink. Besides, the excess of DMSO on the electrode surface can interact with amino group of antibody, which decreases the Mab hCG immobilization efficiency. The sensitivity of both sensors will be examined in more detail below.

\section{Impedance spectra of hCG antibody-antigen interaction}

To evaluate the interaction between Mab hCG and $\alpha$-hCG, the modified electrodes of immunosensor $\mathrm{A}$ and $\mathrm{B}$ are exposed to various concentration of $\alpha$-hCG. The corresponding Nyquist plots of impedance spectra for both immunosensors are shown in (Figure 4). The results show that the diameter of the Nyquist semicircle increases with increasing of $a$-hCG concentration. This could be due to the binding of more antigen molecules to immobilization Mab hCG at higher concentration of antigen. Therefore, the interfacial charge transfer was hindered significantly, resulting in a corresponding increase in the charge transfer resistance. Besides, as the concentration of $\alpha$-hCG increases, the surface coverage must ultimately become saturate because all antibodies on the electrode surface will have already bound with hCG antigen. This can be seen in (Figure 4b) (for immunosensor B), where the impedance spectrum changes only slightly as the $\alpha$-hCG concentration is increased from 15 to $30 \mathrm{ng} / \mathrm{mL}$. However, in the case of immunosensor $\mathrm{A}$, the significant change in the impedance spectrum is still observed as the $a-h C G$ concentration increases up to $70 \mathrm{ng} / \mathrm{mL}$ (Figure $4 \mathrm{a}$ ). This result was further confirmed that the Mab hCG immobilization efficiency of the immunosensor B is higher than immunosensor A's.

The fitting impedance parameters are given in (Table 1 ) and 2 for immunosensor A and B, respectively. The obtained impedance spectra of immunosensor A are well fit by the Randles equivalent circuit at frequencies higher than $165 \mathrm{mHz}$. The impedance behavior at frequencies less than $165 \mathrm{mHz}$ is unexpected and is currently unknown. As can be seen from these tables, the charge transfer resistance $\mathrm{R}_{\mathrm{CT}}$ increases with increasing of hCG concentration. The normalized percentage change of the $\mathrm{R}_{\mathrm{CT}}$ values ranging from $8 \%$ to $39 \%$ for immunosensor $\mathrm{A}$ and from $34 \%$ to $81 \%$ for immunosensor B. This result is demonstrated that the sensitivity of immunosensor B higher than that of immunosensor A. The increase in the $\mathrm{R}_{\mathrm{CT}}$ with increasing protein coverage has been reported $[6,10,23]$ Furthermore, the relative change in $\mathrm{R}_{\mathrm{CT}}$ is much larger than the relative change in Cdl during antigen binding. This is often observed for impedance protein detection $[21,23]$.

The (Figure 5) presents the calibration curve of $R_{C T}$ vs. different concentrations of hCG antigen (C) for both immunosensors. In the 
Citation: Lien TTN, Viet NX, Chikae M, Ukita Y, Takamura Y (2011) Development of Label-Free Impedimetric Hcg-Immunosensor Using ScreenPrinted Electrode. J Biosens Bioelectron 2:107. doi:10.4172/2155-6210.1000107

case of immunosensor A (Figure 5a), a linear range was obtained from 1 to $70 \mathrm{ng} / \mathrm{mL}$ of antigen concentration with the linear equation of $\mathrm{R}_{\mathrm{CT}}$ $=99.8+0.7 \times \mathrm{C}(\mathrm{ng} / \mathrm{mL})$. However, we observed two linear parts in the calibration curve of immunosensor $\mathrm{B}$ (Figure $5 \mathrm{~b}$ ). The $\mathrm{R}_{\mathrm{CT}}$ increased slowly with increasing of antigen concentration from $2 \mathrm{pg} / \mathrm{mL}$ to 2 $\mathrm{ng} / \mathrm{mL}$ and speed-up in the range from 2 to $30 \mathrm{ng} / \mathrm{mL}$. As mentioned above, the $\mathrm{R}_{\mathrm{CT}}$ value denotes the blocking behavior of electrode surface for redox probe. The phenomenon of blocking electrode surface is due to space occupying of huge antigen molecules when they bind with small antibody molecules on the electrode surface. At high concentration of antigen, the competition of hCG antigen to occupy the space leads to the dramatically increase of $\mathrm{R}_{\mathrm{CT}}$. This is the reason why the calibration curve exhibits two linear parts. The obtained linear equation in the range from $2 \mathrm{pg} / \mathrm{mL}$ to $2 \mathrm{ng} / \mathrm{mL}$ for this sensor is $\mathrm{R}_{\mathrm{CT}}=1.25+1.69$ $\times \log \mathrm{C}(\mathrm{pg} / \mathrm{mL})$. Based on the standard deviation of blank sample and slope of calibration curve, the detection limit (LOD) can be calculated as

$$
L O D=\frac{3 x S T D E V}{\text { slope }}
$$

The detection limit (LOD) of immunosensor $\mathrm{A}$ and $\mathrm{B}$ is determined to be $12 \mathrm{ng} / \mathrm{mL}$ and $33 \mathrm{pg} / \mathrm{mL}$, respectively. This result was further confirmed the sensitivity of immunosensor B higher than that of immunosensor A. Furthermore, this experimental result also exposed that the designed immunosensor B is more sensitive than other previously reported immunosensors in the case of detection limit and linear range for antigen detection $[1,2,7,13]$.

\begin{tabular}{|c|c|c|c|}
\hline \multirow{2}{*}{$\begin{array}{c}\mathrm{hCG} \\
(\mathrm{ng} / \mathrm{mL})\end{array}$} & \multicolumn{3}{|c|}{ Equivalent circuit elements for nonfaradaic interface } \\
\cline { 2 - 4 } & $\mathrm{R}_{\mathrm{CT}}(\mathrm{k} \Omega)$ & $\mathrm{C}_{\mathrm{dl}}(\mu \mathrm{F})$ & $\mathrm{R}_{\mathrm{s}}(\mathrm{k} \Omega)$ \\
\hline Blank & $90.47 \pm 5.42$ & $2.55 \pm 0.15$ & $1.54 \pm 0.04$ \\
\hline 1 & $98.24 \pm 5.97$ & $1.75 \pm 0.12$ & $1.50 \pm 0.07$ \\
\hline 5 & $98.37 \pm 1.24$ & $1.59 \pm 0.09$ & $1.59 \pm 0.03$ \\
\hline 10 & $106.57 \pm 3.49$ & $1.66 \pm 0.08$ & $1.51 \pm 0.09$ \\
\hline 20 & $111.40 \pm 3.90$ & $1.48 \pm 0.10$ & $1.57 \pm 0.04$ \\
\hline 30 & $116.40 \pm 3.90$ & $1.64 \pm 0.05$ & $1.55 \pm 0.07$ \\
\hline 40 & $129.47 \pm 0.31$ & $1.69 \pm 0.09$ & $1.53 \pm 0.07$ \\
\hline 50 & $132.93 \pm 0.40$ & $1.51 \pm 0.10$ & $1.52 \pm 0.07$ \\
\hline 70 & $148.87 \pm 5.28$ & $1.25 \pm 0.12$ & $1.61 \pm 0.05$ \\
\hline
\end{tabular}

Table 1: Impedance parameters were obtained from the equivalent circuit fit to the impedance spectra of immunosensor A, which is presented in Figure 4a.

\begin{tabular}{|c|c|c|c|}
\hline \multirow{2}{*}{$\begin{array}{c}\mathrm{hCG} \\
(\mathrm{ng} / \mathrm{mL})\end{array}$} & \multicolumn{3}{|c|}{ Equivalent circuit elements for nonfaradaic interface } \\
\cline { 2 - 4 } & $\mathrm{R}_{\mathrm{CT}}(\mathrm{k} \Omega)$ & $\mathrm{C}_{\mathrm{dl}}(\mu \mathrm{F})$ & $\mathrm{R}_{\mathrm{s}}(\mathrm{k} \Omega)$ \\
\hline Blank & $3.27 \pm 0.18$ & $3.16 \pm 0.18$ & $5.98 \pm 0.12$ \\
\hline 0.2 & $4.97 \pm 0.20$ & $2.78 \pm 0.18$ & $6.02 \pm 0.11$ \\
\hline 0.3 & $5.68 \pm 0.26$ & $2.76 \pm 0.07$ & $6.03 \pm 0.13$ \\
\hline 0.5 & $5.88 \pm 0.20$ & $2.69 \pm 0.08$ & $6.02 \pm 0.12$ \\
\hline 1.0 & $6.26 \pm 0.17$ & $2.89 \pm 0.14$ & $6.02 \pm 0.15$ \\
\hline 2.0 & $6.78 \pm 0.32$ & $2.69 \pm 0.11$ & $6.03 \pm 0.12$ \\
\hline 3.0 & $7.88 \pm 0.22$ & $2.92 \pm 0.18$ & $5.99 \pm 0.11$ \\
\hline 4.0 & $9.82 \pm 0.48$ & $3.13 \pm 0.17$ & $6.05 \pm 0.14$ \\
\hline 5.0 & $10.86 \pm 0.59$ & $2.69 \pm 0.13$ & $6.10 \pm 0.18$ \\
\hline 7.0 & $12.02 \pm 0.51$ & $2.78 \pm 0.16$ & $6.06 \pm 0.11$ \\
\hline 10 & $14.15 \pm 0.82$ & $2.47 \pm 0.10$ & $6.02 \pm 0.12$ \\
\hline 15 & $14.84 \pm 1.85$ & $2.54 \pm 0.19$ & $5.99 \pm 0.13$ \\
\hline 20 & $15.85 \pm 1.20$ & $2.57 \pm 0.11$ & $6.05 \pm 0.13$ \\
\hline 30 & $16.99 \pm 1.41$ & $2.58 \pm 0.06$ & $5.99 \pm 0.13$ \\
\hline
\end{tabular}

Table 2: Impedance parameters were obtained from the equivalent circuit fit to the impedance spectra of immunosensor $B$, which is illustrated in Figure $4 \mathrm{~b}$.
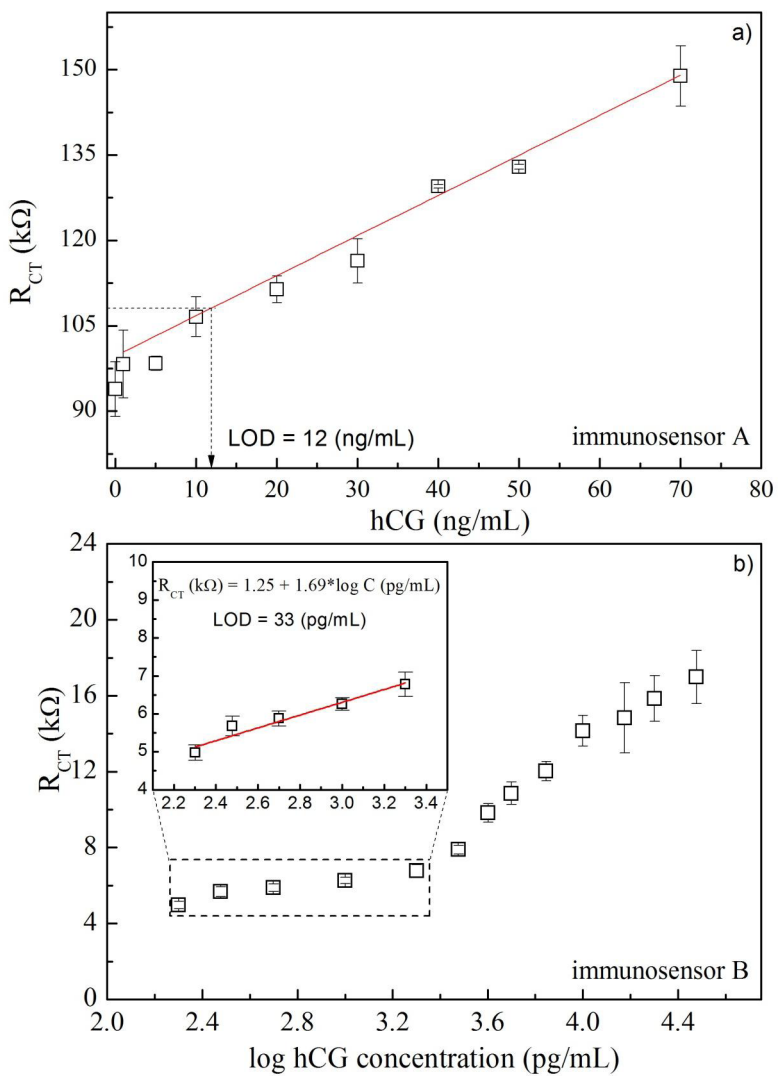

Figure 5: The calibration curves obtained from immunosensor $A$ and $B$ using charge transfer resistance $R_{\mathrm{CT}}$ as function of $\alpha-h C G$ concentration $C$. All data points are average values for the responses of three electrodes and error bars indicatethe standard error

\section{Conclusion}

The results presented in this work concern successful implementation of a simple and specific approach for hCG antibody immobilization onto carbon surface of DEP chip using functional molecule, 1-pyrenebutanoic acid, succinimidyl ester. The versatility of this simple approach could be applied to other biological molecules. Additional information, the experimental results exposed that the designed immunosensor B is more sensitive than other previously reported immunosensors in the case of detection limit and linear range for antigen detection. Moreover, the used of inexpensive DEP chip as a basis for these immunosensors will allow simple instrumentation, disposable and portable at low cost. Besides, based on the current study, it was found that EIS is an impressive method for monitoring the interaction of antigen with antibody that occurred on the electrode surface. This method used in this work can also be applied to other immune systems.

\section{Acknowledgements}

Dr. Truong TN Lien gratefully acknowledge the receipt of a grant from the Japan Society for the Promotion of Science (JSPS) which enabled them to carry out this work.

\section{References}

1. Ramanavicius A, Finkelsteinas A, Cesiulis H, Ramanavicience A (2010) Electrochemical impedance spectroscopy of polypyrrole based electrochemical immunosensors. Bioelectrochem 79: 11-16. 
Citation: Lien TTN, Viet NX, Chikae M, Ukita Y, Takamura Y (2011) Development of Label-Free Impedimetric Hcg-Immunosensor Using ScreenPrinted Electrode. J Biosens Bioelectron 2:107. doi:10.4172/2155-6210.1000107

2. Rodriguez A, Valera E, Ramon-Azcon J, Sanchez FJ, Marco MP, et al. (2008) Sens Actuators B 129: 921-928.

3. Silva BVM, Cavalcanti IT, Mattos AB, Moura P, Sotomayor MDPT, et al. (2010) Disposable immunosensor for human cardiac troponin T based on streptavidinmicrosphere modified screen-printed electrode. Biosens and Bioelectron 26: 1062-1067.

4. Bard AJ, Faulkner LR (1980). New York: John Wiley \& Sons 316-367.

5. Pejcic B, Marco RD (2006) Impedance spectroscopy: Over 35 years of electrochemical sensor optimization.

6. Li CM, Chen W, Yang X, Sun CQ, Gao C, et al. (2005) Impedance labelless detection-based polypyrrole protein biosensor. Front Biosci 10: 2518-2526.

7. Esseghaier C, Helali S, Fredj HB, Tlili A, Abdelghani A (2008) Polypyrrole-neutravidin layer for impedimetric biosensor. Sens Actuators B Chem 131: 584-589.

8. Zhang D, Peng Y, Qi H, Gao Q, Zhang C (2010) Label-free electrochemical DNA biosensor array for simultaneous detection of the HIV-1 and HIV-2 oligonucleotides incorporating different hairpin-DNA probes and redox indicator. Biosens Bioelectron 25: 1088-1094.

9. Katz E (1994) Application of bifunctional reagents for immobilization of proteins on a carbon electrode surface: Oriented immobilization of photosynthetic reaction centers. J Electroanal Chem 365: 157-164

10. Tsekenis G, Garifallou GZ, Davis F, Millner PA, Gibson TD, et al. (2008) Labeless Immunosensor Assay for Myelin Basic Protein based upon an AC Impedance Protocol Anal Chem 80: 2058-2062.

11. Jaegfeldt H, Kuwana T, Johansson G (1983) Electrochemical stability of catechols with a pyrene side chain strongly adsorbed on graphite electrodes for catalytic oxidation of dihydronicotinamide adenine dinucleotide. J Am Chem Soc 105: $1805-1814$

12. Daniel JS, Pourmand N (2007) Label-Free Impedance Biosensors: Opportunities and Challenges. Electroanal 19: 1239-1257.
13. Hafaid I, Chebil S, Youssoufi K, Bessueille F, Errachid A et al. (2010) Effect of electrical conditions on an impedimetric immunosensor based on a modified conducting polypyrrole. Sens. Actuators B 144: 323-331.

14. Jiang J, Basu M, Seggerson S, Miller A, Pugia M, et al. (2007) Nanomaterials for biosensors. Wiley-VCH publishing 208-236

15. Rogers KR (2006) Recent advances in biosensor techniques for environmental monitoring. Anal Chim Acta 568: 222-231.

16. Kerman K, Nagatani N, Chikae M, Yuhi T, Takamura Y, et al. (2006) Label-Free Electrochemical Immunoassay for the Detection of Human Chorionic Gonadotropin Hormone. Anal Chem 78: 5612-5616.

17. Yang L, Li Y (2005) AFM and impedance spectroscopy characterization of the immobilization of antibodies on indium-tin oxide electrode through self-assembled monolayer of epoxysilane and their capture of Escherichia coli O157:H7. Biosens Bioelectron 20: 1407-1416.

18. Tudorache M, Bala C (2007) Biosensors based on screen-printing technology, and their applications in environmental and food analysis. Anal Bioanal Chem 388: 565-578.

19. Chen RJ, Zhang Y, Wang D, Dai H (2001) J Am Chem Soc 123: 3838-3839.

20. Ionescu RE, Gondran C, Bouffier L, Renault NJ, Martelet C, et al. (2010) Labelfree impedimetric immunosensor for sensitive detection of atrazine. Electrochimica Acta 55: 6228-6232.

21. Suni II (2008) Trends Anal Chem 27: 604-610.

22. Shih WC, Yang MC, Lin MS (2009) Development of disposable lipid biosenso for the determination of total cholesterol. Biosens and Bioelectron 24: 16791684 .

23. Huang Y, Bell MC, Suni II (2008) Impedance Biosensor for Peanut Protein Ara h 1. Anal Chem 80: 9157-9161. 